\title{
Accretion Processes in CV AM CVn SDSS J090221.35+381941.9
}

\author{
M.M. Montgomery ${ }^{* \dagger}$ \\ University of Central Florida Physics Department \\ E-mail: montgomerydphysics.ucf.edul
}

SDSS J090221.35+381941.9 is an Cataclysmic Variable AM CVn system that has three most likely evolutionary tracks as defined by the donor type - the hydrogen star channel, the white dwarf channel, and the helium star channel. To break the degeneracy, we numerically model this system three different ways, one for each channel, keeping the mass transfer rate, orbital period, and secondary-to-primary mass ratio constant. Because the white dwarf channel produced a shorter Stage A positive superhump period than in Stage B of the superoutburst, which has never been observed in CV dwarf novae systems, the white dwarf channel has been eliminated as the a likely contender. From a comparison of the system's parameters with the other two evolutionary channels, we find that the helium star channel is most likely for this system and the donor is non-degenerate. As the mass transfer rate is so low, the donor may be starting its evolution into a hybrid white dwarf and migrating from the helium star channel to the white dwarf channel, where it will join its older sibling GP Com.

Accretion Processes in Cosmic Sources - APCS2016 -

5-10 September 2016,

Saint Petersburg, Russia

\footnotetext{
* Speaker.

$\dagger$ I would like to thank the organizers of this committee for inviting me to present this work.
} 


\section{Introduction}

AM CVn systems are very close binaries and thus short orbital period (i.e., $5<P_{\text {orb }}<65$ minute) systems. They form by three most likely evolutionary channels, all of which require the primary to have evolved through the red giant stage, to the white dwarf stage. If after the first common envelope phase, the primary white dwarf is in such a wide orbit with a main sequence secondary that another common envelope phase is not likely, then the system typically evolves as a normal hydrogen $(\mathrm{H})$ Cataclysmic Variable $(\mathrm{CV})$ system. If, however, the $\sim 1 M_{\odot}$ secondary donor fills its Roche lobe at a time when its hydrogen abundance in its core has a fraction $<0.4$ (see e.g., Solheim 2010 and references within), the system may evolve to a long-period AM CVn system by magnetic braking and gravitational wave emission. This channel is known as the hydrogen star (a.k.a, CV) channel. Although not discussed in Solheim (2010), an end result that cannot be ruled out for this track is a supernova type Ia (SN Ia).

If after the first common envelope phase the binary is not wide and the mass of the unevolved secondary star is $M_{2} \leq 2.3 M_{\odot}$ after leaving the second common envelope phase, then the donor can evolve into another helium (He) white dwarf. This double degenerate AM CVn system has both a white dwarf primary and a white dwarf secondary and thus is known as the white dwarf channel.

If after the first common envelope phase the binary is not wide and the mass of the unevolved secondary star is $2.3 \leq M_{2} \leq 5 M_{\odot}$, then the secondary has a non-degenerate helium core after leaving the second common envelope phase. As the star converts helium into carbon in its core, the donor becomes semi-degenerate. Meanwhile, emission of gravitational waves brings the primary and secondary closer together, eventually initiating mass transfer from the donor to the primary, forming an AM CVn system. This channel is known as the helium star channel.

A potential exists for the helium star channel to merge with the white dwarf channel if the helium in the core of the secondary is exhausted before Roche lobe overflow and mass transfer begins. If this happens, then the secondary becomes a hybrid white dwarf, merging with and then continuing on the white dwarf channel. One difference between the white dwarf donors in the white dwarf channel and the hybrid white dwarfs from the helium star channel is mass: The hybrid white dwarf has the larger mass.

Once the AM CVn system has formed, it is categorized in Solheim (2010) into one of four groups: If $P_{\text {orb }}<12$ minutes, then the AM CVn system does not have an accretion disk. If $12<$ $P_{\text {orb }}<20$ minutes, then the accretion disk is large and stable in a superoutburst state, akin to that in a novalike $\mathrm{CV}$ system. If $20<P_{\text {orb }}<40$ minutes, then the accretion disk is variable in size as the system goes through outbursts and superoutbursts, the latter of which is brighter and lasts longer than outbursts and shows hump-shaped (a.k.a., superhump) modulations in its light curve like those in CV SU UMa systems. Lastly, if $P_{\text {orb }}>40$ minutes, then the accretion disk is small and stable, akin to that in a $\mathrm{CV}$ dwarf novae in quiescence.

End results of AM CVn systems are poorly studied. However, two are discussed in Solheim (2010): One proposed end result for both the white dwarf channel and the helium star channel is a SN 1a or a weaker supernova event. A second, more likely, end result is a cold white dwarf in a close binary with a brown dwarf mass object (but not a brown dwarf, per se) or a planet mass object (but not a planet, per se) that may eventually tidally disrupt, generating a debris disk that pollutes the white dwarf. To know which is more likely requires more studies of AM CVn systems. 
In this work, we aim to determine the most likely evolutionary channel for AM CVn SDSS J090221.35+381941.9 (herein, J0902), which has an orbital period of $48.31 \pm 0.08$ minutes as found by Rau et al. (2010) via spectroscopy. According to the orbital period categorizations of Solheim (2010), J0902 should have a small, stable accretion disk in quiescence. However, on March 1516, 2014, J0902 entered into a superoutburst, thereby suggesting a review of the orbital period ranges to now accommodate AM CVn J0902. During this superoutburst, positive superhumps have been observed with periods $P_{+A}=0.03409$ (1) days for the rise to superoutburst Stage A and $P_{+B}=0.03371$ (1) days for the subsequent plateau of the superoutburst Stage B. From analytical models involving the positive superhump period and orbital period data from Stage A only, Kato et al. (2014) finds a mass ratio $q=M_{2} / M_{1}=0.041$ (7) for J0902. As shown in Figure 1 by the thick black vertical line crossing the thin black, red, and blue lines, any of the three AM CVn evolutionary tracks (i.e., white dwarf, hydrogen rich CV, or helium star) are possible. To break the degeneracy, the mass transfer rate of J0902 is needed, which requires knowledge of the masses and radii of the objects in the system as well as the mass-radius relation of the donor.

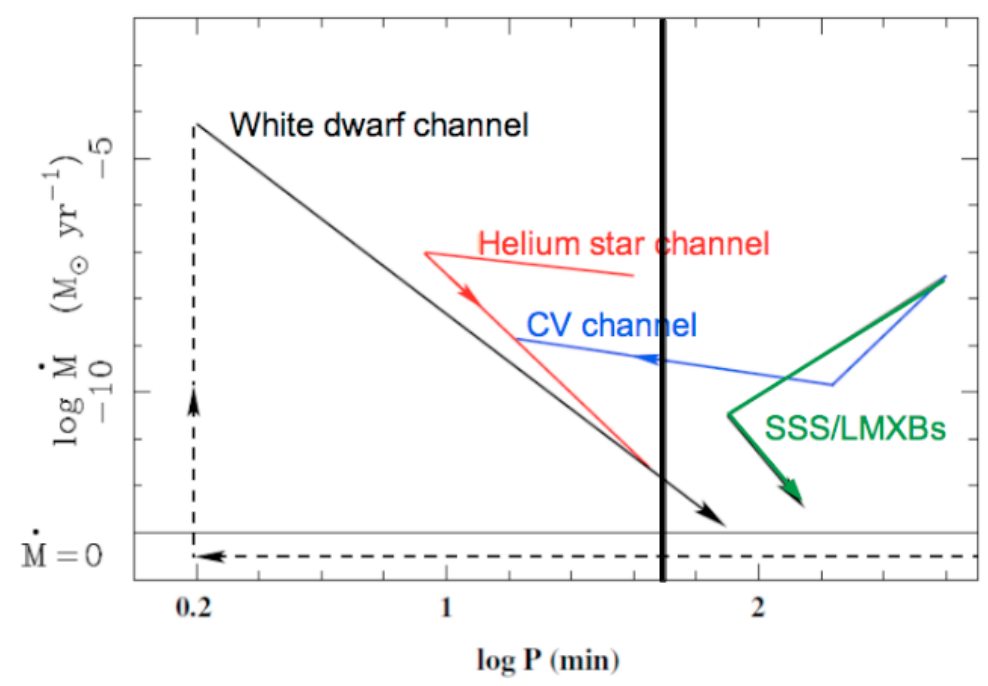

Figure 1: Log-log scale of orbital period versus mass transfer rate. The black dashed line indicates the detached phase of the white dwarf channel whereas the thin solid black line indicates the evolution of the AM CVn channel. The helium star channel is colored red and the hydrogen star (CV) channel is colored blue. In comparison, the evolution of a hydrogen-rich CV such as an SU UMa or a low mass X-ray binary system is colored green. The heavy vertical black line indicates the orbital period of J0902.

To see if variables other than masses, radii, orbital period, and mass transfer rate could shed light on a most-likely or least-likely evolutionary channel, we numerical simulated J0902 three different ways, one for each possible channel show in Figure 1. Since mass transfer rate and the generation of positive superhunps are highly dependent upon masses in the AM CVn system, we aim to see if positive superhump period can also be a parameter that could indicate a most- or least-likely evolutionary channel. We also aim to find the most likely channel for J0902. In §2, we summarize our numerical study, and in $\S 3$, we compare our findings with that of others to determine the likely channel for J0902. In $\S 4$, we provide conclusions. 


\section{Numerical Simulations - A Summary}

In Montgomery (2017), we numerically simulate Stage A and Stage B of a superoutburst in J0902 to induce positive superhumps in the simulated lightcurve. The numerical code is 3D Lagrangian method of Smoothed Particle Hydrodynamics (SPH) as reviewed in e.g., Montgomery (2012a,b). It assumes an ideal gas law of low adiabatic gamma and a low alpha in the $\alpha$-disc model of Shakura \& Sunyaev (1973). The numerical code generates a geometrically thin disk from scratch and particles interact with a fixed smoothing length of $h \sim 0.02$. We assume 25,000 particles, which is a number that is high enough to reproduce the positive superhumps in the light curve, yet low enough for computational purposes.

As noted in the previous section, parameters important for determining the evolutionary track are mass transfer rate, $P_{o r b}$, and the separation distance $a$; for mass transfer rate, we need the primary and secondary masses, or mass ratio $q=M_{2} / M_{1}$, and the donor mass-radius $M_{2}-R_{2}$ relation. One equation that relates these parameters is Newton's version of Kepler's Third Law

$$
P_{o r b}^{2}=\frac{4 \pi^{2} a^{3}}{G\left(M_{1}+M_{2}\right)},
$$

where $G$ is the Universal Gravitational Constant. Another relation involves the radius of the secondary,

$$
\frac{R_{2}}{a}=\frac{0.49 q^{2 / 3}}{0.6 q^{2 / 3}+\ln \left(1+q^{1 / 3}\right)},
$$

which is accurate to better than $1 \%$ and $\mathrm{s}$ good for all $q$. A third is the donor's mass-radius relation. Since we don't know the evolutionary channel for J0902, we modify the code three different ways, one for each of the three channels. For the CV hydrogen star channel, we chose the relation

$$
R_{2}=\left(\frac{M_{2}}{M_{\odot}}\right)^{13 / 15} R_{\odot}
$$

which is a reasonable approximation for $0.08 \leq M_{2} \leq 1.0$. For the white dwarf channel, we chose the relation

$$
R_{2}=0.0155\left(\frac{M_{2}}{M_{\odot}}\right)^{-0.212} R_{\odot} .
$$

from Armstrong et al. (2012) and references within. For the helium star channel, we chose the relation

$$
R_{2}=0.029\left(\frac{M_{2}}{M_{\odot}}\right)^{-0.19} R_{\odot}
$$

from Armstrong et al. (2012) and references within. Using $q=0.041$ (7) (Kato et al. 2014) and $P_{\text {orb }}=48.31 \pm 0.08$ minutes (Rau et al. 2010), we solve for the other parameters. The list of solved and assumed parameters is listed in Table 1. A very similar table appears in Montgomery (2016).

Each of the three simulations generates Stage A and Stage B positive superhumps in the simulated light curves. Table 2 lists the simulated positive superhump periods for each simulated stage and each evolutionary channel. As shown in Table 2, the white dwarf channel produces a simulated Stage A positive superhump period that is shorter than that of Stage B, which has not been observed to date in SU UMa CV systems (Kato \& Osaki, 2013). As such, this channel is eliminated as a possibility for J0902 as discussed in Montgomery (2017). 
Table 1: Solved and Assumed Input Parameters for Potential Channels of J0902

\begin{tabular}{lccccc}
$\begin{array}{l}\text { Evolutionary } \\
\text { Channel }\end{array}$ & $\begin{array}{c}\mathrm{M}_{1} \\
\left(\mathrm{M}_{\odot}\right)\end{array}$ & $\begin{array}{c}\mathrm{M}_{2} \\
\left(\mathrm{M}_{\odot}\right)\end{array}$ & $\begin{array}{c}a \\
\left(\mathrm{R}_{\odot}\right)\end{array}$ & $\begin{array}{c}P_{\text {orb }} \\
(\mathrm{min})\end{array}$ & $\begin{array}{c}R_{2} \\
\left(\mathrm{R}_{\odot}\right)\end{array}$ \\
\hline CV H star & 1.24 & 0.0508 & 0.477 & 48.30 & 0.0756 \\
White Dwarf & 0.216 & 0.00886 & 0.266 & 48.23 & 0.164 \\
He Star & 0.586 & 0.0240 & 0.372 & 48.28 & 0.0589 \\
\hline
\end{tabular}

Table 2: Simulated $P_{+}$Periods for Possible J0902 Evolutionary Channels

\begin{tabular}{lcc} 
Simulated Channel & $\begin{array}{c}\text { Stage A } \\
\mathrm{P}_{+} \text {(days) }\end{array}$ & $\begin{array}{c}\text { Stage B } \\
\mathrm{P}_{+} \text {(days) }\end{array}$ \\
\hline CV H Star Channel & 0.03433 & 0.03398 \\
White Dwarf Channel & 0.03390 & 0.03395 \\
He Star Channel & 0.03400 & 0.03395 \\
\hline
\end{tabular}

\section{The Likely Channel for J0902}

As shown in Table 2, both the CV hydrogen star channel and the helium star channel produce a general trend of decreasing positive superhump period from Stage A to Stage B that mimics the general trend in the observational periods. Thus, both channels remain contenders for evolution of J0902. Let's look at each channel more closely, starting with the helium star channel: As discussed in Solheim (2010), once the donor's mass reduces to $\sim 0.01-0.03 M_{\odot}$ and the orbital period has grown to $P_{\text {orb }} \gtrsim 35-40$ minutes, the donor may develop into a fully degenerate cooling white dwarf. The helium star channel and the white dwarf channel merge, as discussed in $\S 1$ and as shown in Figure 1, and the donor evolves into a hybrid white dwarf as it migrates from the helium star channel to the white dwarf channel. Compared to the donor mass and orbital period for the helium star channel in Table 1, the donor in J0902 may be a hybrid white dwarf. Figure 2 shows a comparison of donor mass to orbital period for various AM CVn systems and general regions where the least-to-most evolved helium star donors would be located. As shown, J0902 and GP Com sharing the same region of the graph, which is where the helium star and white dwarf channels merge. As we can't definitely determine the evolutionary channel from this figure, we turn to the donor mass-radius relations and the donor mass.

Figure 3 shows secondary mass-radius relations for various AM CVn systems as well as for the helium star donors (upper solid curve) and the fully degenerate white dwarf donors (lower solid curve). For $\log \left(M_{2}\right)>-1.6$, the heavy dash-dot curve roughly defines the boarder between the white dwarf donors and the helium star donors. As shown in Figure 3, J0902 shares a similar mass-radius relation to that of GP Com, assuming the He star channel values for mass and radius in Table 1. Figure 3 suggests the helium star channel for J0902 and the donor is non-degenerate.

Now let's look at the hydrogen CV star channel. As Figure 1 and Table 2 suggest that either the CV star or helium star channel is possible, the deciding factor may be the chemistry. As discussed in Solheim (2010), a H/He ratio of $10^{-3}$ could indicate the hydrogen star channel whereas a $\mathrm{H} / \mathrm{He}$ 


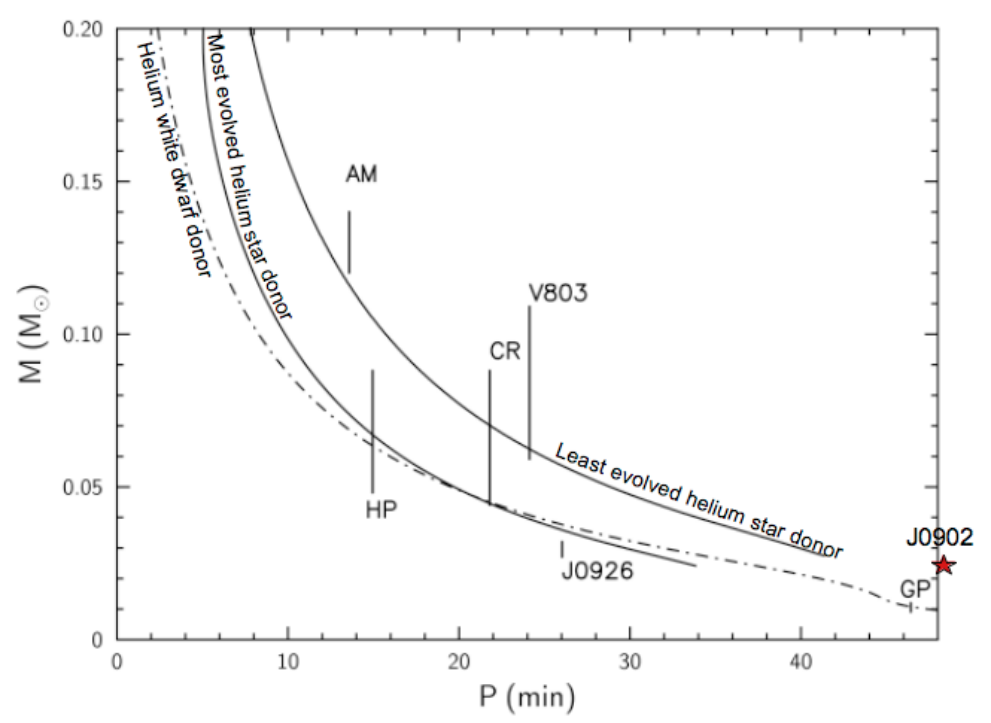

Figure 2: Orbital period versus donor mass shows a comparison of AM CVn systems GP Com (GP), SDSS J092638.71+362402.4 (J0926), V803 Cen (V803), CR Boo (CR), HP Lib (HP), and AM CVn (AM). Solid curves represent $P_{\text {orb }}-M_{2}$ relations for least (i.e., non-degenerate) helium stars (top) and fully degenerate helium donors (bottom), and the dash-dot curve represents a partially degenerate helium white dwarf donor. Figure is modified from Solheim (2012) to include J0902 (star).

ratio of $10^{-5}$ could indicate the helium star channel. As Rau et al. (2010) did not find hydrogen in the spectra of J0902, the hydrogen star channel is highly unlikely for J0902.

Returning to Figure 1, if J0902 is evolving on the helium star channel, then its current mass transfer rate is approximately $10^{-12} M_{\odot} / y r$. This low rate is similar to that listed in Table 2 of Solheim (2010) for J0902 and is similar to the mass transfer rate of GP Com. Possibly J0902 is a younger sibling to GP Com: As the accretion rate is so low, possibly the donor in J0902 is just starting to evolve into a hybrid white dwarf that will eventually evolve on the white dwarf channel with GP Com.

\section{Conclusions}

In this work, we review the simulations of three possible evolutionary channels that J0902 may populate. From the numerical simulation trends in the generation of positive superhump periods from Stage A to Stage B, the white dwarf channel does not seem possible for J0902. A lack of hydrogen in the spectra suggests that J0902 is not a contender for the hydrogen star channel. Secondary mass-radius relations suggest that the helium star channel is likely for J0902 and the donor is non-degenerate, assuming the helium star donor mass and radius values used in the 3D SPH simulation of J0902. The orbital period and donor mass suggest that the donor may be evolving into a hybrid white dwarf as it migrates from the helium star channel to the white dwarf channel. 


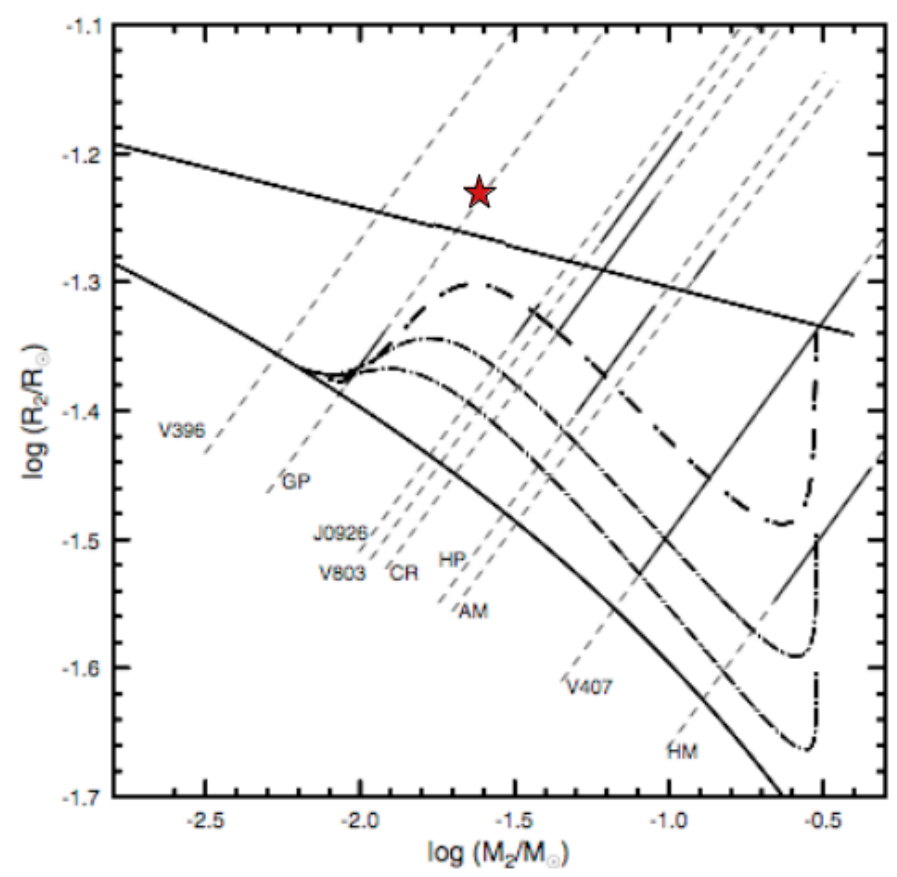

Figure 3: Log-log plot shows $M_{2}-R_{2}$ relations - dash lines denote various AM CVn systems that fill their Roche lobes and solid line over plots indicate estimated masses; upper and lower solid curves denote helium star donors and $\mathrm{T}=0 \mathrm{~K}$ helium white dwarf (i.e., fully degenerate) donors, respectively; heavy dash-dot curve denotes a mildly degenerate white dwarf donor whereas the thin dash-dot curves from top-to-bottom denote increasing degeneracy. Figure is modified from Solheim (2012) to include J0902 (star).

\section{References}

[1] Armstrong, E., Patterson, J., \& Kemp, J., 2012, MNRAS, 421, 2310

[2] Kato, T., \& Osaki, Y., 2013, PASJ, 65, 115

[3] Montgomery, M.M., 2012, ApJ, 745, 25

[4] Montgomery, M.M., 2012, ApJ, 735, 27

[5] Montgomery M.M., 2017, ASPC, 509, 549

[6] Montgomery M.M., 2017, NewA, submitted.

[7] Rau, A., Roelofs, G.H.A., Groot, P.J., et al. 2010, ApJ, 708, 456

[8] Shakura, S., \& Sunyaev, R.A., 1973, A\&A, 149, 135

[9] Solheim J.-E., 2010, PASP, 122, 1133 